\title{
Massive macroglossia, a rare side effect of COVID-19: clinical, histologic, and genomic findings in COVID-19-positive versus COVID-19-negative patients
}

\author{
Victoria A. Mañón ${ }^{1} \cdot$ David Chubb ${ }^{1}$ Laura S. Farach ${ }^{2} \cdot$ Rachid Karam $^{3} \cdot$ Mary C. Farach-Carson ${ }^{4}$. \\ Nadarajah Vigneswaran $^{4} \cdot$ Karan Saluja $^{5} \cdot$ Simon Young ${ }^{1} \cdot$ Mark Wong $^{1} \cdot$ James C. Melville $^{6}$
}

Received: 13 October 2021 / Accepted: 10 December 2021 / Published online: 3 January 2022

(c) The Author(s), under exclusive licence to Springer-Verlag GmbH Germany, part of Springer Nature 2022

\begin{abstract}
Purpose The primary purpose of this study is to identify if there is an underlying genetic predisposition for COVID-related macroglossia and if this susceptibility is higher among individuals of African heritage. Secondary objectives include determining if genetic testing of COVID-infected patients who are intubated and prone could identify patients with higher susceptibility to the development of macroglossia.

Methods A retrospective chart review was completed for each patient, and prospectively, genetic and histopathologic analyses were completed. Whole-exome sequencing was completed on two patients; immunohistochemistry was completed on the COVID-positive tissue samples.

Results Histopathology of the COVID-positive patient revealed significant peri-lymphocytic infiltrate, which was absent in the COVID-negative patient. Immunohistochemistry confirmed the presence of immune cells. Results from the wholeexome sequencing were inconclusive.

Conclusion The findings of this study are consistent with others that have observed a lymphocytic infiltrate in the organs of patients infected with SARS-CoV-2. On histology, IHC highlighted a CD45 + predominance, indicating that a robust immune response is present in the tissues. The pathobiology of this phenomenon and its role in the development and/or persistence of massive macroglossia requires further study.
\end{abstract}

Keywords Oral pathology $\cdot$ COVID-19 $\cdot$ Oral surgery $\cdot$ Histopathology $\cdot$ Immunohistochemistry

Victoria A. Mañón

Victoria.A.Manon@uth.tmc.edu

1 Bernard and Gloria Pepper Katz Department of Oral \& Maxillofacial Surgery, The University of Texas at Houston, TX 77054 Houston, USA

2 Department of Pediatrics, McGovern Medical School, The University of Texas Health Science Center, Houston, USA

3 Department of Research and Development, Ambry Genetics, Aliso Viejo, CA 92656, USA

4 Department of Diagnostic and Biomedical Sciences, The University of Texas at Houston, Houston, USA

5 Department of Pathology and Laboratory Medicine, The University of Texas Health Science Center, Houston, USA

6 Head and Neck Oncologic and Microvascular Reconstructive Surgery, Bernard and Gloria Pepper Katz Department of Oral \& Maxillofacial Surgery Department of Oral \& Maxillofacial Surgery, The University of Texas at Houston, Houston, USA

\section{Summary}

In this investigator-initiated study, two patients, one COVIDpositive and one COVID-negative, with macroglossia secondary to prolonged intubation in the prone position were evaluated via histopathologic and genetic analysis. The histopathology for the COVID-positive and COVID-negative patients varied significantly, with peri-vascular and perilymphatic infiltrate seen in the lingual tissues of the COVIDpositive specimens; immunohistochemistry was completed. These features were absent in the COVID-negative patient's histology. Whole-exome sequencing (WES) was negative for pathologic variants in genes clinically associated with macroglossia. 


\section{Introduction}

Macroglossia is defined as the protrusion of the tongue beyond the teeth in a natural resting posture. ${ }^{1}$ Acute macroglossia is a rare medical complication and can range in severity; the exact etiology is largely unknown. Proposed etiologies of acute macroglossia include trauma and medication side effects, including the use of ACE inhibitors, allergic reactions, pressure packing of the oropharynx, oral surgery, and neurogenic causes. ${ }^{2-10}$ The initial, acute onset of macroglossia is hypothesized to be a form of compartment syndrome in the tongue. ${ }^{11}$ Compression or obstruction of the lingual arteries, veins, and lymphatic system leads to tongue edema. Mechanical compression during intubation and anesthesia may lead to macroglossia via compression of arteries and/or veins, particularly in lengthy surgical cases or prolonged intubation. , $^{2,7,10}$ The number of COVID-19 patients now being treated with prone positioning has drastically increased. ${ }^{12}$ We hypothesize this will lead to an increased number of macroglossia cases, particularly among African American patients. At present, the authors are aware of seven cases of COVID + patients who were intubated and developed non-resolving massive macroglossia. Of the seven patients, six are Black.

In COVID-positive patients, peri-vascular and perilymphocytic infiltration may be a contributing factor to the development and persistence of macroglossia. Inflammatory cell infiltration with myocyte injury has been reported and is associated with fatal COVID-19 infections. ${ }^{13}$ The presence of inflammatory infiltrate on H\&E staining in COVID-positive patients is consistent with findings seen in the cardiac tissue from previous reports. Another possible etiology of acute macroglossia progressing to chronic massive macroglossia refractory to non-surgical therapy may be prolonged intubation in the prone position in select individuals with a genetic predisposition. Of the cases observed by this team, all patients but one were African American; the other patient was Caucasian. Of the observed patients, two elected and consented to participate in the study, limiting the analysis. One patient was COVID positive and the other was COVID negative. The role of the ACE2 receptor in both SARS-CoV2 infection and hypertension indicates that prior use of ACE inhibitors may also contribute to the development of angioedema. The purpose of this study is to compare the histopathology of two patients with massive macroglossia and attempt to identify a potential underlying genetic predisposition for COVID-related macroglossia in African American patients.

\section{Methods}

This study was an investigator-initiated, combination retrospective and prospective case series involving two patients with a history of massive macroglossia. One COVID-negative and one COVID-positive patient were analyzed. Retrospectively, a chart review was completed for each patient, and prospectively, genetic and histopathologic analyses were completed. Both consented to have their blood and saliva used for genomic analysis. Immunohistochemistry (IHC) was performed on formalin-fixed paraffin-embedded 3 - $\mu \mathrm{m}$ sections using fully automated systems ("Dako Omnis with Envision FLEX detection system"; Agilent Technologies, CA, USA and "intelliPATH with MACH detection"; Biocare Medical LLC, CA, USA) and following pre-diluted ready-to-use antibodies: CD45 (clone 2B11 + PD7/26, Agilent), CD3 (polyclonal, Agilent), CD4 (clone 4B12, Agilent), CD8 (clone C8/144B, Agilent), CD20cy (clone L26, Agilent), FoxP3 (clone 236A/E7, Biocare), and anti-human podoplanin (clone D2-40, Agilent). Positive and negative controls were used throughout. Clinical whole-exome sequencing was completed through a CLIA laboratory (Ambry Genetics, CA) and analyzed through their clinical bioinformatics process, as previously described. ${ }^{14}$ In addition, a filtered variant list was generated for our review. The list consisted of benign and inconclusive variants, as well as heterozygous deleterious variants in autosomal recessive disorders. All variants were reviewed for association with macroglossia or a similar process, using the OMIM database (omim. org). The variants were assessed for similarities between the patients. The study was approved by the UTHealth Institutional Review Board.

\section{Results}

\section{Patients}

Patient 1 (Fig. 1A) is a 46-year-old African American male from Florida who was hospitalized for approximately 3 months due to COVID-19 infection-related complications. During his hospital stay, he was intubated and placed on a ventilator in the prone position for 21 days. While in the prone position, he developed severe macroglossia. Although the patient recovered from the COVID-19 infection, the macroglossia resulted in both airway compromise and decreased oral intake necessitating a tracheostomy and percutaneous endoscopic gastrostomy (PEG) tube. The patient was refractory to non-surgical treatment of the macroglossia, including compression therapy. He was 
Fig. 1 A and B Clinical photos of patient 1 and patient 2, respectively. Both patients demonstrate persistent, massive macroglossia
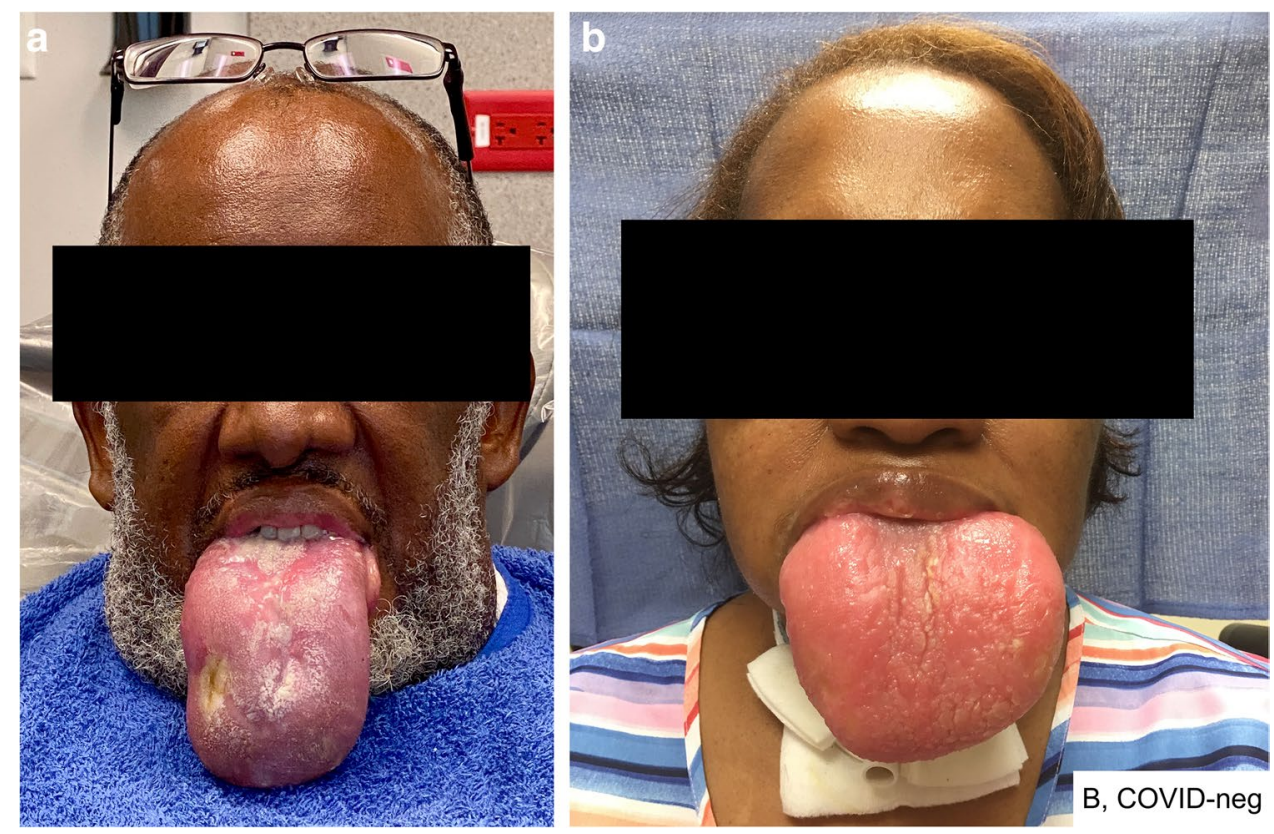

then referred to the Oral and Maxillofacial (OMS) department at the University of Texas Health Science Center at Houston for surgical management.

Patient 2 (Fig. 1B) is a 48-year-old African American female who was hospitalized following a cerebrovascular accident. During her hospital stay, she required intubation for 14 days. Extubation was complicated by angioedema and macroglossia following the administration of an ACE inhibitor. Due to airway compromise, the patient required a tracheostomy and PEG placement. The patient's macroglossia was also refractory to conservative measures and was subsequently referred to the OMS department at the University of Texas Health Science Center at Houston.

\section{Gross and histopathology findings}

The gross evaluation showed a $9.2 \times 7.1 \times 2.5 \mathrm{~cm}$ hemi-glossectomy specimen (patient 1 ) with focal white plaques on its surface (Fig. 2A). Sectioning showed a homogeneous, edematous, glistening cut surface with focal fibrosis without distinct mass formation. Histologic sections showed mild hyperkeratotic mucosal surface with no dysplasia. Submucosal tissue was edematous with splaying of skeletal muscle fibers, with focal fibrosis and hemosiderin-laden macrophages, and moderate peri-vascular and peri-lymphatic chronic inflammation composed primarily of small mature lymphocytes, scattered plasma cells and mast cells, and few eosinophils (Fig. 2B). The focal germinal center formation was noted within the lymphoid aggregates. IHC panel was performed which highlighted predominantly CD45 + lymphocytes (Fig. 2C), of which $\sim 85 \%$ were CD3 + T-cells (Fig. 2D) and $\sim 15 \%$ were CD20 + B-cells (Fig. 2E). Of the CD3 + T-lymphocytes, $90 \%$ were CD4 + helper T-cells (CD4/CD3) (Fig. 2F) and 10\% were CD8 + cytotoxic T-cells (CD8/CD3) (Fig. 2G). Forkhead box P3 (FoxP3) stain highlighted $\sim 10 \%$ of lymphocytes were CD4 +-regulatory T-cells of CD4 + T-helper cells in the lymphoid aggregates (FoxP3/CD4) (Fig. 2H). D2-40 stain highlighted endothelial cells of the lymphatic and vascular spaces within the lymphoid aggregates. Congo-red cytochemical stain was negative for amyloid deposition.

\section{Genetic analysis}

In both patients, whole-exome sequencing was negative for pathogenic variants in genes clinically associated with macroglossia. Patients 1 and 2 had 61 and 78 filtered variants; respectively. Patient 1 had a heterozygous variant in IDUA associated with Hurler syndrome, an autosomal recessive disorder which can present with an enlarged tongue, along with multi-system effects, not observed in the patient. The variant was predicted tolerated and only one variant was observed. Patient 2 had no variants associated with macroglossia. The patients had variants in 4 common genes (CCDC78, CUBN, G6PD, TTN) but the variants were not associated with macroglossia or inflammatory infiltrate, and all but one was predicted tolerated.

\section{Discussion}

The etiology of massive, persistent macroglossia is multifactorial and has multiple proposed etiologies. Macroglossia has been noted to be caused by genetic disorders, trauma, 
Fig. 2 A Gross examination showed a $9.2 \times 7.1 \times 2.5 \mathrm{~cm}$ hemi-glossectomy specimen with focal white plaques and hemorrhage on its dorsal surface. B Low-power histologic section show mild submucosa edema with peri-lymphatic and peri-vascular chronic inflammation comprising of predominantly of small mature lymphocytes ((hematoxylin and eosin, total magnification $(\mathrm{TM}) \times 40$ ), inset $\mathrm{TM} \times 200$ ); $\mathbf{C}$ immunoshistochemical (IHC) stain show CD45 + lymphocytes in the lymphoid aggregates $(\mathrm{TM} \times 100)$, inset $(\mathrm{TX} \times 200) ; \mathbf{D}$ and $\mathbf{E}$ IHC stains show majority $(\sim 85 \%)$ of the CD45+lymphocytes are CD3 + T-cells (CD3, D $\mathrm{TM} \times 100$, inset $(\mathrm{TM} \times 200)$ and $15 \%$ of the CD45 + lymphocytes are $\mathrm{CD} 20+\mathrm{B}$-cells (CD20, E TM $\times 100$, inset $(\mathrm{TM} \times 200) ; \mathbf{F}$ and $\mathbf{G}$ IHC stains show of the CD3 + T-lymphocytes, majority $(90 \%)$ of them are CD4 + T-helper cells $(\mathrm{CD} 4, \mathbf{F} \mathrm{TM} \times 100$, inset $(\mathrm{TM} \times 200))$ and $10 \%$ are CD8 + cytotoxic T-cells (CD8, G TM $\times 100$, inset $(\mathrm{TM} \times 200)$; H FOXP3 IHC stain show of the CD4 + T-helper cells, 10\% cells are $\mathrm{CD} 4+$-regulatory $\mathrm{T}$-cells in the lymphoid aggregates $(\mathrm{FOXP} 3, \mathbf{H} \mathrm{TM} \times 100$, inset $(\mathrm{TM} \times 200))$
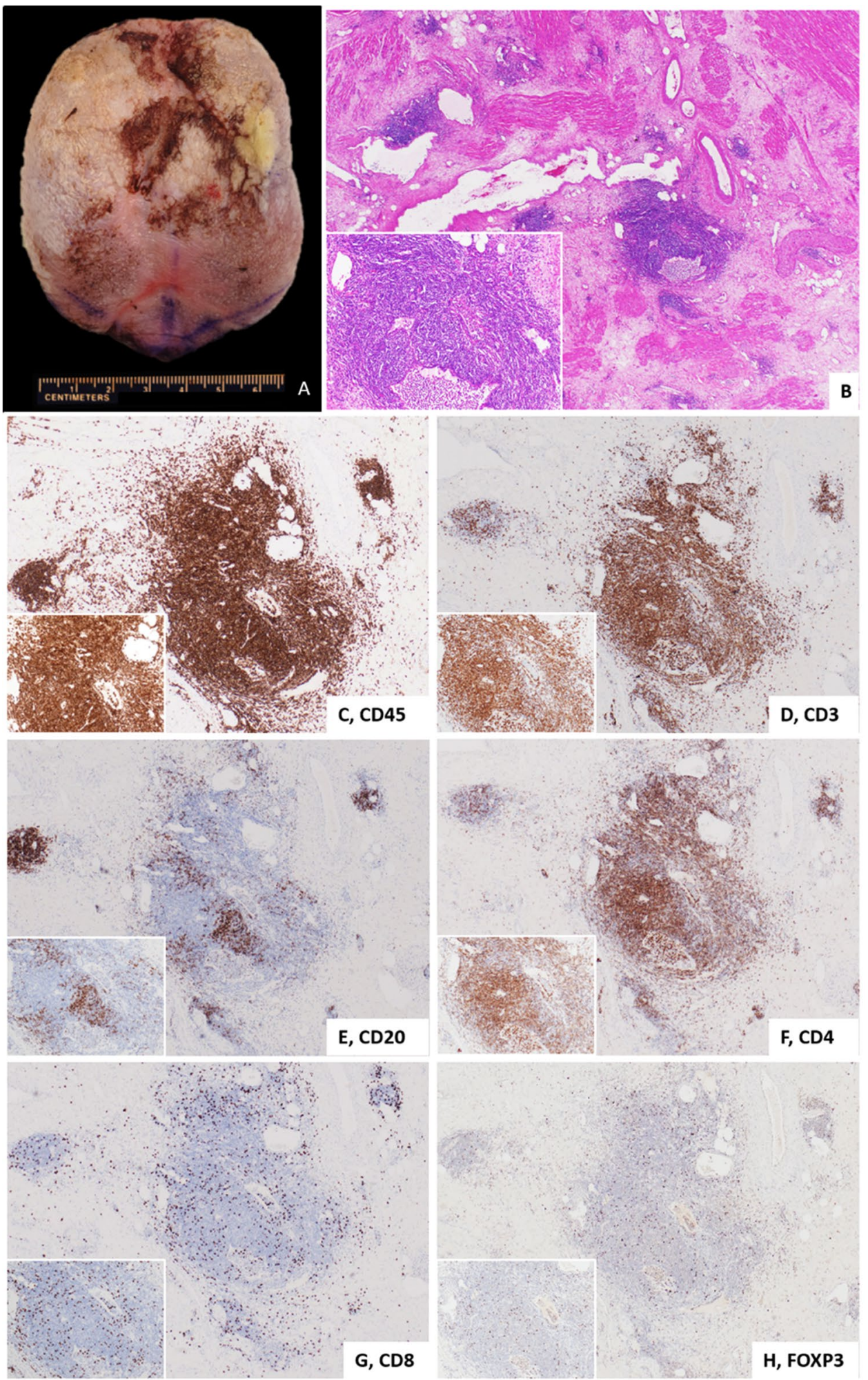

F, CD4

H, FOXP3 lymphedema, medication side effects, and/or other metabolic derangements. This article presents two patients with similar clinical presentations of persistent massive macroglossia after prolonged intubation in the prone position. Despite their clinical similarities, these two cases demonstrated strikingly different histological features. One primary clinical difference between patient 1 and patient 2 is infection with SARS-CoV-2; the patient infected with SARS-CoV-2 had significant lymphocytic infiltrate on histology that was not observed in the histology from the COVID-negative 
patient. These histological differences indicate that the pathogenesis of this condition differs for each patient, with a strong immune response secondary to COVID-19 infection being an important factor in the pathogenesis of macroglossia development and/or persistence for the COVID-positive patient.

Infection with COVID-19 appears to be an inciting factor for inflammatory cell infiltrates. The results of the IHC panel indicate that the SARS-CoV-2 virus may be causing a significant immune reaction that generates a significant T-cell response, as the panel highlighted a CD3 + lymphocytic predominance in the tissues. This phenomenon has also been seen in cardiac and renal tissues of patients infected with COVID-19. ${ }^{13,15}$ In cardiac tissues, the presence of SARS-CoV-2 was associated with increased densities of lymphocytes and macrophages. These findings correlated clinically to the patient's disease severity, cytokine storm, and increased mortality. Additionally, the patients' existing medical conditions did not contribute to the cardiac inflammation, suggesting that the inflammation was not pre-existing and was caused by the induced immune response. The authors of these studies also found evidence of the viral RNA in the liver and kidneys, indicating that the virus has a multi-organ presence. ${ }^{13,15}$ It was also noted that patients that were previously taking immunosuppressants, primarily glucocorticoids, had less severe myocarditis and less myocardial inflammation as compared to those not on immunosuppressant therapy. Glucocorticoids dampen the immune response by suppressing T-cell function, which would explain this finding in cardiac myocytes. ${ }^{16}$.

The findings in the cardiac tissues are consistent with our findings in the lingual tissues of the COVID-positive patient. The IHC panel highlighted predominantly CD45+ lymphocytes, of which $\sim 85 \%$ were CD3 + T-cells and $\sim 15 \%$ were CD20+B-cells. This indicates a strong immune response secondary to the viral infection, which was absent in the COVID-negative specimen. These differences indicate that the pathogenesis of macroglossia in patients with COVID-19 infection may be propagated and/or sustained by induction of a strong immune response and inflammation secondary to viral infection. Further studies would be required to determine the pathogenesis of SARS-CoV-2 and its induction of the immune response in tissues. While angioedema secondary to venous/lymphatic compression is likely a contributing factor to macroglossia in the COVID-positive patient, the presence of immune cells is a striking finding and may have implications for prognosis as seen in other organ systems.

In the COVID-negative patient, vascular compression from prolonged intubation in the prone position is the likely etiology. Although rare, persistent massive macroglossia has been observed in other patients that are COVID-negative and remained intubated for prolonged periods of time. ${ }^{2}$ While this patient required surgical resection, conservative management (i.e., use of compression dressings) resolves less severe cases. ${ }^{17}$ At this time, clinical markers that may indicate a successful response to conservative therapy have yet to be determined. As observed in this study, genetic variants that may indicate a genetic predisposition to developing macroglossia were not identified. More studies are needed to determine which patient factors may indicate susceptibility for the development of massive, persistent macroglossia.

In conclusion, the findings of this study are consistent with others that have observed a lymphocytic infiltrate in the organs of patients infected with SARS-CoV-2. On histology, IHC highlighted a CD45 + predominance, indicating that a robust immune response is present in the tissues. The pathobiology of this phenomenon and its role in the development and/or persistence of massive macroglossia requires further study. While pathological variants were not identified from WES, the limited sample size does not rule out genetic predisposition as a possible etiology for macroglossia.

Acknowledgements The authors thank Meghan Town, MS, CGC, LGC, at Ambry Genetics for her invaluable help coordinating the receipt and analysis of the samples.

Author contribution Victoria A. Mañón, DDS MBA: Contributed to design, data acquisition and interpretation, drafted and critically revised the manuscript

David Chubb BDS: Contributed to design, data acquisition, and drafted the manuscript

Laura S. Farach MD: Contributed to design, data acquisition and interpretation, and drafted the manuscript

Rachid Karam PhD: Contributed to design, data interpretation, and critically revised the manuscript

Mary C. Farach-Carson PhD: Contributed to design, data interpretation, and critically revised the manuscript.

Nadarajah Vigneswaran BDS DrMedDent DMD: Contributed to conception and design, data interpretation, and critically revised the manuscript

Karan Saluja MD: Contributed to design, data interpretation, drafted and critically revised the manuscript

Simon Young DDS MD PhD FACS: Contributed to conception and design, data interpretation, and critically revised the manuscript

Mark Wong DDS FACS: Contributed to conception and design, data interpretation, and critically revised the manuscript

James C. Melville DDS FACS: Contributed to conception and design, data interpretation, and critically revised the manuscript

All authors gave their final approval and agree to be accountable for all aspects of the work.

Funding Supported by the Hinds-Helfrick grant funding from the University of Texas Health Science Center, Bernard \& Gloria Pepper Katz Department of Oral \& Maxillofacial Surgery, and Ambry Genetics.

Ethical approval.

IRB approval was sought and obtained from the University of Texas Health Science Center at Houston.

\section{Declarations}

Informed consent All patients in this study were informed and signed consent forms indicating their understanding and participation in the study. 
Conflict of interest The authors declare no competing interests.

\section{References}

1. Prada CE, Zarate YA, Hopkin RJ: Genetic causes of macroglossia: diagnostic approach. Pediatrics 129:e431, 2012

2. Andrews E, Lezotte J, Ackerman AM: Lingual compression for acute macroglossia in a COVID-19 positive patient. BMJ Case Rep 13:e237108, 2020

3. Bozkurt S, Arslan ED, Köse A, Ayrık C, Yılmaz A, Dündar GA (2015) Lingual angioedema after alteplase treatment in a patient with acute ischemic stroke. World J Emerg Med 6:74

4. DePasse JM, Palumbo MA, Haque M, Eberson CP, Daniels AH (2015) Complications associated with prone positioning in elective spinal surgery. World journal of orthopedics 6:351

5. Gadban H, Talmon Y, Gilbey P, Samet A (2003) Acute edema of the tongue: a life-threatening condition. Ann Otol Rhinol Laryngol 112:651

6. Kawaguchi M, Sakamoto T, Ohnishi H, Karasawa J (1995) Pharyngeal packs can cause massive swelling of the tongue after neurosurgical procedures. Anesthesiology 83:434

7. Miura Y, Mimatsu K, Iwata H (1996) Massive tongue swelling as a complication after spinal surgery. J Spinal Disord 9:339

8. Norman JL, Holmes WL, Bell WA, Finks SW (2013) Life-threatening ACE inhibitor-induced angioedema after eleven years on lisinopril. J Pharm Pract 26:382

9. Saah D, Braverman I, Elidan J, Nageris B (1993) Traumatic macroglossia. Ann Otol Rhinol Laryngol 102:729

10. Sinha A, Agarwal A, Gaur A, Pandey CK (2001) Oropharyngeal swelling and macroglossia after cervical spine surgery in the prone position. J Neurosurg Anesthesiol 13:237

11. Lam AM, Vavilala MS (2000) Macroglossia: compartment syndrome of the tongue? Anesthesiology 92:1832

12. Mittermaier M, Pickerodt P, Kurth F, de Jarcy LB, Uhrig A, Garcia C, Machleidt F, Pergantis P, Weber S, Li Y, Breitbart A,
Bremer F, Knape P, Dewey M, Doellinger F, Weber-Carstens S, Slutsky AS, Kuebler WM, Suttorp N, Müller-Redetzky H: Evaluation of PEEP and prone positioning in early COVID-19 ARDS. EClinicalMedicine 28:100579, 2020

13. Bearse M, Hung YP, Krauson AJ et al (2021) Factors associated with myocardial SARS-CoV-2 infection, myocarditis, and cardiac inflammation in patients with COVID-19. Mod Pathol 34:1345-1357. https://doi.org/10.1038/s41379-021-00790-1

14. Farwell KD, Shahmirzadi L, El-Khechen D, Powis Z, Chao EC, Tippin Davis B, Baxter RM, Zeng W, Mroske C, Parra MC, Gandomi SK, Lu I, Li X, Lu H, Lu HM, Salvador D, Ruble D, Lao M, Fischbach S, Wen J, Lee S, Elliott A, Dunlop CL, Tang S (2015) Enhanced utility of family-centered diagnostic exome sequencing with inheritance model-based analysis: results from 500 unselected families with undiagnosed genetic conditions. Genet Med 17(7):578-586. https://doi.org/10.1038/gim.2014.154 (Epub 2014 Nov 13 PMID: 25356970)

15. Jacobs W, Lammens M, Kerckhofs A, Voets E, Van San E, Van Coillie S, Peleman C, Mergeay M, Sirimsi S, Matheeussen V, Jansens H, Baar I, Vanden Berghe T, Jorens PG (2020) Fatal lymphocytic cardiac damage in coronavirus disease 2019 (COVID19): autopsy reveals a ferroptosis signature. ESC Heart Failure 7:3772-3781. https://doi.org/10.1002/ehf2.12958

16. Davis TE, Kis-Toth K, Szanto A, Tsokos GC (2013) Glucocorticoids suppress $\mathrm{T}$ cell function by up-regulating microRNA-98. Arthritis Rheum 65(7):1882-1890. https://doi.org/10.1002/art. 37966

17. Klosterman T, Tatum SA (2015) Current surgical management of macroglossia. Curr Opin Otolaryngol Head Neck Surg 23:302

Publisher's Note Springer Nature remains neutral with regard to jurisdictional claims in published maps and institutional affiliations. 